RESEARCH PAPER

\title{
Estimating the health consequences of replacing cigarettes with nicotine inhalers
}

\section{W Sumner II}

Background: A fast acting, clean nicotine delivery system might substantially displace cigarettes. Public health consequences would depend on the subsequent prevalence of nicotine use, hazards of delivery systems, and intrinsic hazards of nicotine.

Methods: A spreadsheet program, DEMANDS, estimates differences in expected mortality, adjusted for nicotine delivery system features and prevalence of nicotine use, by extending the data and methods of the SAMMEC 3 software from the US Centers for Disease Control and Prevention. The user estimates disease risks attributable to nicotine, other smoke components, and risk factors that coexist with smoking. The public health consequences of a widely used clean nicotine inhaler replacing cigarettes

Correspondence to: Walton Sumner II, Department of Medicine, Division of General Medical Sciences, Box 8005, 660 South Euclid Avenue, St Louis, MO 63110, USA wsumner@im.wustl.edu

Received 17 January 2002. Accepted 6 February 2003 were compared to historical observations and public health goals, using four different risk attribution scenarios and nicotine use prevalence from 0-100\%.

Main outcome measures: Changes in years of potential life before age 85 (YPL85).

Results: If nicotine accounts for less than a third of smokers' excess risk of SAMMEC diseases, as it most likely does, then even with very widespread use of clean nicotine DEMANDS predicts public health gains, relative to current tobacco use. Public health benefits accruing from a widely used clean nicotine inhaler probably equal or exceed the benefits of achieving Healthy People 2010 goals.

Conclusions: Clean nicotine inhalers might improve public health as much as any feasible tobacco control effort. Although the relevant risk estimates are somewhat uncertain, partial nicotine deregulation deserves consideration as part of a broad tobacco control policy.
V arious observations suggest that the health risks associated with cigarette smoking are infrequently caused by nicotine, the addictive ingredient. ${ }^{12}$ Other components of smoke are likely contributors to smoking related disease. Cigarette smoke carries thousands of reactive chemicals, including many known toxins and carcinogens. ${ }^{3}$ Tobacco control advocates often cite these as ample reason to quit smoking. In contrast, nicotine itself seems to be relatively safe. ${ }^{1}$ Smoking cessation counsellors increasingly encourage long courses of nicotine to help injured smokers and special populations. ${ }^{45}$ Certainly, nicotine replacement is safer than smoking.

Potential reduced exposure products (PREPs) are nicotine delivery systems designed to expose users to fewer toxins than cigarettes do. Examples include smokeless tobacco, heated tobacco devices, and pharmaceutical nicotine replacement therapies. The Institute of Medicine report, Clearing the smoke: assessing the science base for tobacco harm reduction, described how little we know about the health consequences of PREPs in general. ${ }^{2}$ The report suggested a broad, detailed research effort to improve our understanding of the consequences of chronic PREP use. These results would inform future PREP and tobacco regulation. However, the results of such research would not be available for many years. Furthermore, different PREP designs may have diverse health consequences.

Meanwhile, pharmaceutical nicotine products receive much greater scrutiny from the US government than either the traditional or novel tobacco products they might displace. These clean nicotine products deliver pharmaceutical grade nicotine without exposing the user to any other known or suspected harmful chemicals. While tobacco companies introduce new tobacco products without review, pharmaceutical companies face significant regulatory obstacles at every step in the development of clean nicotine products. The discrepancy causes some authors to call for a "level playing field" in which regulators tolerate or even encourage some alternatives to cigarettes. $^{5-10}$ Reversal of this historical accident requires increased regulation of tobacco products, reduced regulation of clean nicotine products, or both. Increased regulation of tobacco products is costly and controversial, and therefore will wax and wane with political and economic fortunes. Reduced regulation of clean nicotine could lead to the development of delivery systems designed to establish or maintain nicotine addiction. If people believe these products to be safer than cigarettes, then nicotine use may increase. Some smokers would switch nicotine sources rather than quit. ${ }^{68}$ Some ex-smokers and never-smokers might become regular users, exposing these groups to the health hazards of nicotine. Injuries accruing to never-smokers and potential ex-smokers who use clean nicotine might offset illness avoided in smokers who switch to safer nicotine sources. The net public health consequences are uncertain. However, Kozlowski and colleagues ${ }^{4}$ offer as an example that habitual medicinal nicotine use might expose the user to a lifetime mortal risk of only 1 in 10000 . If so, then public health would improve even if everyone used a clean nicotine product, as long as it displaced cigarettes.

Although delivering nicotine without other smoke constituents should significantly reduce harms to current smokers, efforts to cleanse tobacco smoke at the point of use have failed to benefit public health. ${ }^{11}$ The cigarette industry responded to health concerns by shifting to filtered, lower tar cigarettes between 1950 and 1970. ${ }^{12}$ The US National Cancer Institute later attempted to develop a safe cigarette by removing harmful constituents from cigarette smoke, ${ }^{13}$ but never developed a

Abbreviations: DEMANDS, Differences in Expected Mortality Adjusted for Nicotine Delivery Systems; PREP, potential reduced exposure products; SAMMEC, Smoking Attributable Morbidity, Mortality and Economic Costs; YPLG, years of potential life gained; YPLL, years of potential life lost 
viable product. The RJ Reynolds-Nabisco company tested two nearly smokeless heated tobacco devices, ${ }^{14}$ and Philip Morris has developed a novel tobacco product to deliver low doses of nicotine. ${ }^{2}$ No tobacco product has yet removed all offensive components of tobacco smoke.

Existing nicotine replacement products have not displaced cigarettes in the market to supply chronic nicotine users. ${ }^{15}$ The probability of long term use of currently available pure nicotine products is relatively low. ${ }^{16}$ Polacrilex gum, transdermal patches, nasal spray, and shallow inhalers ${ }^{17}$ produce much lower and slower peak arterial nicotine concentrations than cigarettes, and may have annoying side effects. A deeply inhaled nicotine powder or aerosol could supply nicotine to the arterial circulation as efficiently as a cigarette, but would maintain nicotine dependence rather than facilitate smoking cessation. A PREP delivering essentially pure nicotine by deep inhalation may be called a clean nicotine inhaler. One prototype for a clean nicotine inhaler has been developed, tested, and patented, but is not being produced commercially. ${ }^{18}$ Other designs might reuse the powder inhaler devices that deliver corticosteroids to patients with chronic lung diseases. Legislative and regulatory constraints increase the time and expense of commercial development, or limit or prohibit marketing, as the history of the nicotine nasal inhaler demonstrates. ${ }^{10}$

Although some observers might welcome a competitive market to supply chronic nicotine users, ${ }^{6}{ }^{19-21}$ others have discouraged chronic nicotine use because of health risks. ${ }^{22}{ }^{23}$ The alternative is to continue vigorous efforts to reduce tobacco use, in the hope of achieving goals such as the Healthy People 2010 target of $12 \%$ smoking prevalence among adults in the USA. ${ }^{24}$ Regulatory debates might benefit from understanding the likely public health consequences of displacing cigarettes with cleaner nicotine delivery systems. The US Centers for Disease Control and Prevention has contributed to tobacco policy debates with its series of SAMMEC (Smoking Attributable Morbidity, Mortality and Economic Costs) computer programs..$^{25-31}$

This paper has two purposes. First, it introduces the DEMANDS program (Differences in Expected Mortality Adjusted for Nicotine Delivery Systems), a spreadsheet combining SAMMEC 3 data and methods with adjustable estimates of risk sources and exposures for the SAMMEC diseases. DEMANDS estimates the public health consequences of arbitrary combinations of cigarette smoking, with or without a competing PREP. Second, this paper contrasts the long term consequences of achieving the Healthy People 2010 goals with an alternative policy in which a clean, fast acting nicotine inhaler completely displaces tobacco cigarettes. Although this analysis highlights some questions about the safety of nicotine and acceptability of a nicotine inhaler, it also suggests that widespread use of clean nicotine inhalers could have tolerable public health consequences.

\section{METHODS \\ Overview}

The DEMANDS program is an adaptation of SAMMEC 3. DEMANDS is limited to predicting changes in years of potential life lost before age 65 (YPLL65) and 85 (YPLL85) resulting from hypothetical shifts in the 1990 US's population nicotine source and historical nicotine use. DEMANDS requires a user to make a number of assertions, including:

(1) fraction of the population smoking cigarettes

(2) fraction of the population using a PREP

(3) PREP nicotine delivery

(4) PREP smoke delivery

(5) smoking harms attributable to nicotine, for each disease

(6) smoking harms attributable to smoke, for each disease

(7) smoking harms attributable to genes and environment, subsequently called correlates (of smoking), for each disease.
From these data DEMANDS estimates relative risks for PREP users, then predicts the number of 1990 deaths that would have occurred in a given scenario.

The current analysis explores the long term consequences of two tobacco control strategies. One strategy reaches the Healthy People 2010 goals for smoking. The second strategy would replace cigarettes with a clean nicotine delivery system. Each strategy is assumed to have established an equilibrium pattern of nicotine use after many years.

\section{DEMANDS software}

The DEMANDS program is a Microsoft Excel spreadsheet extending the mortality predictions of SAMMEC 3. DEMANDS uses most of the data and methods of SAMMEC 3. SAMMEC 3 provides relative risk estimates for 27 tobacco related causes of death, adjusted for age, sex, and smoking status. SAMMEC 3 also provides data on the reported occurrence of deaths from these 27 tobacco related illnesses, and prevalence of smokers and ex-smokers in the USA in 1990. From the prevalence of smoking, relative risks of death from diseases, and absolute numbers of deaths in each disease category, SAMMEC 3 estimates the number of deaths attributable to smoking. It then infers years of potential life lost (YPLL), economic losses, and smoking attributable mortality rates.

DEMANDS predicts years of potential life gained or lost before ages 65 and 85 as a consequence of changes in safety and prevalence of nicotine use, using actual deaths in 1990 as a baseline.

The key assumption in DEMANDS is that four distinguishable features of smoking and smokers cause tobacco related deaths. The first is nicotine, which is present in other nicotine delivery systems. The second is smoke, composed of thousands of gases and particulates. The third is carbon monoxide, distinguished from smoke because some PREPs deliver large quantities of this dangerous gas. The fourth is the collection of all correlates of smoking-for example, characteristics of smokers or ex-smokers that persist whether or not they smoke. Alcoholism, poverty, and psychiatric illness are examples of smoking correlates that could increase mortality from many diseases even if these patients never smoke. Many smokers who die of smoking related illnesses may have had concurrent problems that would have limited the benefits of smoking cessation. DEMANDS therefore requires the data described in the following sections.

\section{Nicotine use}

SAMMEC 3 defines nine populations of tobacco users. There are smokers and ex-smokers, males and females, aged 35-65 years and over 65 years $(2 \times 2 \times 2=8$ combinations $)$, and pregnant smokers. In place of SAMMEC 3's description of the prevalence of smokers and ex-smokers, DEMANDS anticipates 10 nicotine use categories. Dividing each category by sex, age, and pregnancy status yields 50 groups of nicotine users. DEMANDS requires a prevalence estimate for each of these groups. The nicotine use categories and their distinct collections of risks follow.

Categories 1-4 reclassify SAMMEC 3 smokers.

(1) Smokers smoke tobacco cigarettes. Smokers have the relative risks of current smokers. This category includes individuals using both a PREP and cigarettes.

(2) Switchers stop using tobacco in favour of a PREP. Switchers share the correlate risks of smokers; nicotine, smoke, and carbon monoxide risks of the PREP, as applicable; and the smoke and carbon monoxide related risks of ex-smokers if the PREP does not deliver smoke or carbon monoxide.

(3) Would-be smokers have used only the PREP, but would have used tobacco had the PREP been unavailable. Would-be smokers have the correlate risk of smokers, and the risks of the PREP. 
(4) Prohibited smokers would have smoked cigarettes in 1990 , but external events prevent them from ever using any nicotine product, including cigarettes. That is, some combination of taxation, education, social norms, sales restrictions, and smoking prohibitions could have prevented these people, who were smokers in 1990, from even experimenting with tobacco. They have only the correlate risks of smokers. Prohibited smokers (rather than ex-smokers) are required to model the long term reduction in smoking prevalence in the Healthy People 2010 scenario.

Categories 5-8 reclassify SAMMEC 3 ex-smokers.

(5) Ex-smokers stop using tobacco and do not use a PREP. They have the same relative risks as current ex-smokers.

(6) Relapsers are ex-smokers who resume using nicotine when the PREP is available. Relapsers have the correlate risk of ex-smokers, the risks of the PREP, and the smoke and carbon monoxide related risks of ex-smokers if the PREP does not deliver smoke or carbon monoxide.

(7) Would-be ex-smokers have used only the PREP, but would have used and quit tobacco had the PREP been unavailable. Would-be ex-smokers have the correlate risks of ex-smokers and the risks of the PREP.

(8) Prohibited ex-smokers would have stopped using tobacco in 1990, but external events prevent them from ever using any nicotine product. They have only the correlate risks of ex-smokers. In a long term Healthy People scenario, most current ex-smokers are reclassified as prohibited ex-smokers.

Categories 9 and 10 reclassify SAMMEC 3 never-smokers.

(9) New users would never use tobacco products, but do use a PREP. New PREP users experience the risks of the PREP.

(10) Abstainers do not use any nicotine products, and have a relative risk of 1 for SAMMEC diseases.

The current analysis assumes that achievement of Healthy People 2010 goals would include a reduction in smoking initiation, and therefore small numbers of ex-smokers in ensuing decades. The clean nicotine scenarios reclassify all smokers as would-be smokers or prohibited smokers; all exsmokers as would-be ex-smokers or prohibited ex-smokers, and all never-smokers as new users or abstainers. People like current smokers are assumed to use nicotine at lowest prevalence of use. People like current ex-smokers use nicotine if the prevalence is higher, and finally, current never-smokers use nicotine at the highest levels of use.

\section{Nicotine delivery system}

DEMANDS requires estimates of the nicotine, smoke, and carbon monoxide delivery of a new nicotine delivery system, as a multiple of what modern cigarettes deliver. In addition, because SAMMEC 3 simply lists absolute numbers of deaths from fires attributed to cigarettes, DEMANDS requires an estimate of the fire starting propensity of a new PREP, again as a multiple of the hazard posed by cigarettes. These variables would typically vary from zero (no nicotine source) to $100 \%$ (typical cigarettes in 1990) or more. For instance, one might guess that the Eclipse device delivers $90 \%$ of the nicotine, $10 \%$ of the smoke, $130 \%$ of the carbon monoxide, and have $20 \%$ of the fire starting propensity of a traditional cigarette. A clean nicotine inhaler would have $100 \%$ of the nicotine, and $0 \%$ of the smoke, carbon monoxide, and fire starting propensity of cigarettes.

\section{Relative risk fractions}

For each of 26 SAMMEC 3 diseases (excluding burns), DEMANDS requires estimates of the fraction of smokers' excess relative risk that is attributable to one of four general causes of illness: nicotine, carbon monoxide, smoke constituents other than nicotine and carbon monoxide, and smoking correlates. Attributing risks to smoking correlates reduces the estimated benefits of switching nicotine source and smoking cessation relative to continued smoking. Attributing risks to nicotine increases the estimated mortality for ex-smokers and never-smokers who become PREP users, relative to exsmokers and never-smokers. Attributing risk to smoke and carbon monoxide increases the benefits of switching and smoking cessation, without adding risk for ex-smokers and never-smokers who become PREP users. Unlike some PREPs, a clean nicotine inhaler should not deliver carbon monoxide, therefore the current analysis will include carbon monoxide risks with smoke risks

There is no direct evidence for these risk fraction estimates. Therefore, this analysis simply reports a range of possibilities relevant to regulation of a pulmonary inhaler delivering essentially pure nicotine. Four possibilities are considered.

(1) $100 \%$ smoke (all excess relative risk is due to smoke or carbon monoxide)

(2) $85 \%$ smoke, $10 \%$ correlates, $5 \%$ nicotine

(3) $60 \%$ smoke, $30 \%$ correlates, $10 \%$ nicotine

(4) $33 \%$ smoke, $33 \%$ correlates, $33 \%$ nicotine.

An extreme scenario bounds the range of logical possibilities. Although inconsistent with available data, this boundary is useful for estimating other scenarios by rough interpolation.

(5) Mathematical boundary: $100 \%$ nicotine.

\section{Predicted relative risks}

DEMANDS predicts the relative risk of a disease in a population as follows.

(1) For each SAMMEC disease, multiply the estimated fraction of risk attributable to nicotine, smoke, or carbon monoxide with the fraction of that component delivered by the PREP

(2) Take the sum of these products and multiply by the excess risk (that is, the relative risk -1 ) of that disease in the relevant SAMMEC 3 population.

(3) If the PREP does not deliver smoke and the PREP user stops smoking, then multiply the estimated fraction of risk attributable to smoke by the excess risk of the disease in ex-smokers, and add this value to the sum.

\section{(4) Add 1.}

For instance, consider a clean nicotine inhaler delivering $100 \%$ of the nicotine, $0 \%$ of the smoke, and $0 \%$ of the carbon monoxide in a cigarette. Estimate that $10 \%$ of the excess risk of lung cancer is due to nicotine, $60 \%$ to carcinogens in smoke, $0 \%$ from carbon monoxide, and 30\% to correlates. SAMMEC 3 estimates that 35-40 year old male smokers and ex-smokers have relative risks of 22.36 and 9.36, respectively, for developing lung cancer compared to never-smokers. In a smoker who switches to the clean nicotine inhaler, the relative risk of fatal lung cancer falls to:

$$
\begin{aligned}
& 1+\left((1 \times 0.1)_{\text {nicotine }}+(0 \times 0.6)_{\text {smoke }}+(0 \times 0)_{\mathrm{co}}+0.3_{\text {correlate }}\right) \\
& \times(22.36-1)_{\text {smoker excess risk }}+(0.6 \times(9.36-1))_{\text {exsmoker risk }} \\
& 14.56
\end{aligned}
$$

The decline in risk is modest because smoking correlates and nicotine exposure persist, and switchers continue to experience the risks of ex-smokers. For never-smokers who begin using a clean nicotine inhaler, lung cancer risk climbs from 1.0 to $1+(1 \times 0.1) \times(22.36-1)=3.136$.

\section{Death multiplier}

The formula below predicts a multiple of deaths expected from a new pattern of use of a nicotine delivery system, relative to the 1990 experience with cigarette smoking:

$$
\text { Multiple }=\left(\text { Sum }_{\text {for } i=1 \text { to } 10}\left(P_{i}{ }^{*} R R_{i}\right)\right) /\left(P_{n}+P_{s}{ }^{*} R R_{s}+P_{x}{ }^{*} R R_{x}\right)
$$


where $P_{i}=$ expected prevalence of the $\mathrm{i}^{\text {th }}$ DEMANDS class of nicotine users

$\mathrm{RR}_{\mathrm{i}}=$ calculated relative risk of the $\mathrm{i}^{\text {th }}$ DEMANDS class of nicotine users

$\mathrm{P}_{\mathrm{n}}=1990$ prevalence of never-smokers

$\mathrm{P}_{\mathrm{s}}=1990$ prevalence of smokers

$\mathrm{P}_{\mathrm{x}}=1990$ prevalence of ex-smokers

$\mathrm{RR}_{\mathrm{s}}=$ relative risk in smokers

$\mathrm{RR}_{\mathrm{x}}=$ relative risk in ex-smokers

\section{Changes in YPLL}

Multiplying the number of deaths documented in 1990 by the multiplier calculated above yields the number of deaths in a given cohort. The change in deaths in that cohort is the difference between the 1990 observation and the prediction. If the change is positive (the death multiplier is less than 1), then DEMANDS subjects new survivors to general mortality risks in this and ensuing years. DEMANDS uses 1993 estimates of age and sex specific death rates to estimate these deaths. Finally, DEMANDS calculates the difference between observed total YPLL65 and YPLL85 in 1990 from the 27 SAMMEC diseases and total YPLL85 predicted after a change in nicotine use.

\section{Scenarios examined}

The four risk attribution scenarios were combined with steady state nicotine use scenarios. These steady state use scenarios model a constant pattern of nicotine use over many decades, rather than the fluctuating use of cigarettes seen during the 1900s. Clean nicotine inhaler scenarios model use by $0-100 \%$ of adults, in $10 \%$ increments, with no cigarette use so that smokers and ex-smokers are non-existent. The four risk attribution scenarios also were compared to a steady state achievement of the Healthy People 2010 goal of 12\% smoking prevalence, assuming a 5\% prevalence of ex-smokers (again assuming a steady, long established pattern of smoking). The changes in years of potential life lost before age 65 and 85 , relative to actual 1990 experience, were calculated as a function of the prevalence of clean nicotine inhaler use. The years of potential life gained or lost before age 85 , relative to Healthy People 2010 goals, was calculated as a function of the prevalence of clean nicotine inhaler use.

I will refer to reductions in years of potential life lost as "years of potential life gained" (YPLG) to avoid double negative phrases. A positive YPLG is a public health gain, meaning that a year of potential life lost in 1990 would not have been lost in the reported scenario. A negative YPLG is a public health loss, meaning that a year of life that was not lost in 1990 is lost in the scenario.

\section{RESULTS}

\section{Years of potential life gained or lost at steady state}

Figure 1 illustrates the years of potential life gained before age 65 (YPLG 65) as a function of prevalence of use of a clean nicotine inhaler, for four different assumptions about risk attribution. If all excess risk were attributable to smoke rather than nicotine or correlates of smoking, then DEMANDS estimates that all levels of inhaler use yield about one million YPLG 65. Assigning a large amount of risk to nicotine causes net harms with high levels of nicotine use. For example, if two thirds of adults would use a clean nicotine inhaler, and nicotine, smoke, and correlates each accounted for one third of the excess risk of each SAMMEC disease, then there would be no public health benefit to adopting inhalers instead of maintaining 1990 smoking patterns.

Figure 2 provides the same data for YPLG 85. The prevalence of steady state nicotine inhaler use that is neutral for this measure of public health is about $76 \%$, slightly higher than in fig 1.

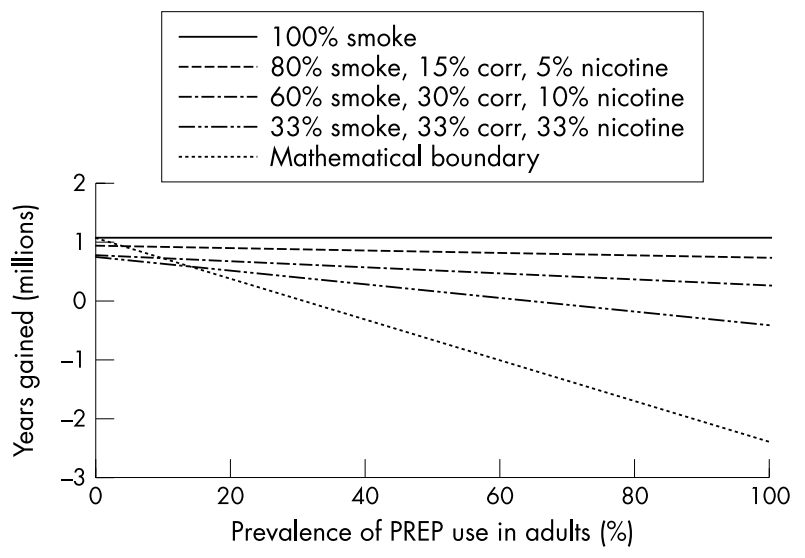

Figure 1 Changes in years of potential life before age 65 if nicotine inhalers displaced cigarettes, relative to the 1990 SAMMEC estimate of YPLL65. Interpolation between the 100\% smoke line and the mathematical limit (hypothetical 100\% of risk from nicotine) yields any risk attribution scenario with $0 \%$ of risk due to correlates. For example, at $100 \%$ prevalence of nicotine use, the vertical distance from the $100 \%$ smoke value to the baseline is about $30 \%$ of the distance from $100 \%$ smoke to the mathematical limit. This indicates that if $100 \%$ of the population uses a clean nicotine inhaler, if nicotine uniformly accounts for $30 \%$ of smoker's excess risk, and if correlates of smoking are irrelevant, then the public health consequences are equivalent to the actual 1990 experience.

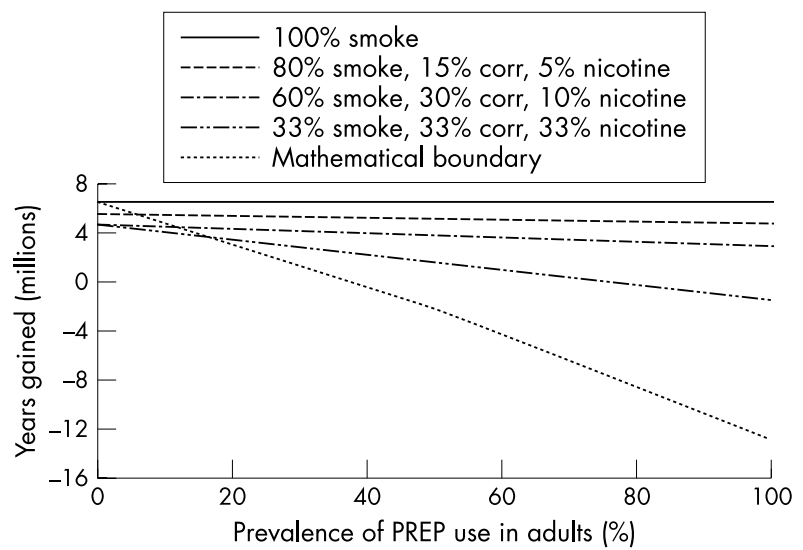

Figure 2 Changes in years of potential life before age 85 if nicotine inhalers displaced cigarettes, relative to the 1990 SAMMEC estimate of YPLL85.

Figure 3 again estimates YPLG 85 for different levels of clean nicotine inhaler use, but assumes steady state Healthy People 2010 goals as the baseline (12\% cigarette smoking prevalence, with 5\% ex-smoking). In each risk scenario, DEMANDS predictions for Healthy People goals in that risk scenario are subtracted from the clean nicotine inhaler predictions, so that 0 on the y axis always represents parity with Healthy People 2010. DEMANDS predictions for Healthy People scenarios (versus 1990) vary from 2-4 million YPLG. When compared to a Healthy People scenario, nicotine inhaler use can be quite prevalent and still yield public health benefits unless nicotine accounts for more than about one third of risk. However, if nicotine users experience less risk, then very widespread inhaler use looks preferable to Healthy People 2010 goals.

\section{Causes of deaths}

Table 1 lists the numbers of deaths DEMANDS predicts for each SAMMEC disease, assuming 50\% PREP prevalence and the four risk scenarios. The overwhelmingly important contributors to premature death are coronary artery disease, 


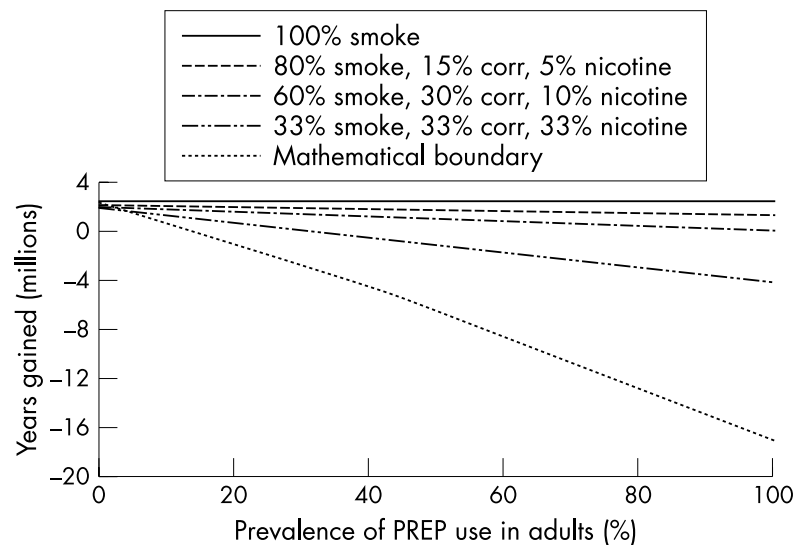

Figure 3 Changes in years of potential life before age 85 if nicotine inhalers displaced cigarettes, relative to the DEMANDS estimate of YPLL85 after achieving Healthy People 2010 goals.

respiratory tract cancer, and chronic obstructive pulmonary disease. Consequently, the most important risk attributions involve these three classifications. Other cardiovascular, infectious, malignant, and childhood diseases and burns contribute far fewer deaths.

One can approximate mixed risk attribution models from table 1. For each SAMMEC disease, select the column that most closely approximates expectations. For example, one might expect that pulmonary diseases are $100 \%$ attributable to smoke; that malignancies are $80 \%$ attributable to smoke, $15 \%$ attributable to correlates, and 5\% attributable to nicotine. The model estimates that $50 \%$ prevalence of clean nicotine use results in 57000 YPLL85 from asthma, compared to 73000 in 1990, yielding a public health gain of 16000 YPLG85 for mortality averted from this disease. Adding gains and losses calculated from the columns that best describe risk attribution for each disease yields an estimate of total change in YPLG85 for $50 \%$ use of a clean nicotine inhaler in the specified risk attribution model, relative to the actual 1990 experience.

\section{DISCUSSION}

The DEMANDS program describes a process for extending SAMMEC methodology and data to estimate the public health consequences of a PREP competing with cigarettes in the marketplace. In the current analysis, DEMANDS was used to estimate the public health consequences of completely displacing cigarettes with a clean nicotine inhaler. Although the correct risk attribution is not known, nicotine probably accounts for much less than a third of smokers' risk for most of the SAMMEC diseases. At this level of risk, displacement of tobacco products with clean nicotine delivery systems should yield public health benefits comparable or superior to the benefits of reaching and maintaining Healthy People 2010 goals in the USA, even if the prevalence of nicotine use in the USA rose

\begin{tabular}{|c|c|c|c|c|c|c|}
\hline \multicolumn{2}{|c|}{ Prevalence } & Actual 1990* & $50 \%$ & $50 \%$ & $50 \%$ & $50 \%$ \\
\hline \multicolumn{2}{|c|}{ Nicotine source } & Cigarettes & Inhaler & Inhaler & Inhaler & Inhaler \\
\hline \multicolumn{2}{|c|}{ Risk from smoke } & NA & 100 & 85 & 60 & 33 \\
\hline \multicolumn{2}{|c|}{ Risk from covariates } & NA & 0 & 15 & 30 & 33 \\
\hline \multicolumn{2}{|c|}{ Risk from nicotine } & NA & 0 & 5 & 10 & 33 \\
\hline \multicolumn{7}{|c|}{ Disease specific YPLL85 ('000s) } \\
\hline $\begin{array}{l}\text { ICD-9 } \\
\text { Tuberculos }\end{array}$ & Disease & & & & & \\
\hline $10-12$ & Respiratory tuberculosis & 22 & 15 & 16 & 18 & 20 \\
\hline \multicolumn{7}{|c|}{ Cancers of: } \\
\hline $140-149$ & Lip, oral cavity, pharynx & 146 & 26 & 52 & 78 & 119 \\
\hline 150 & Oesophagus & 164 & 39 & 65 & 91 & 128 \\
\hline 157 & Pancreas & 342 & 239 & 262 & 285 & 322 \\
\hline 161 & Larynx & 65 & 12 & 23 & 34 & 50 \\
\hline 162 & Trachea, lung, bronchus & 2315 & 339 & 765 & 1192 & 1866 \\
\hline 180 & Cervix uteri & 102 & 72 & 79 & 85 & 96 \\
\hline 188 & Urinary bladder & 104 & 58 & 67 & 76 & 90 \\
\hline 189 & Kidney, other urinary & 160 & 104 & 116 & 128 & 144 \\
\hline \multicolumn{7}{|c|}{ Cardiovascular diseases } \\
\hline $390-398$ & Heart valve disease & 79 & 63 & 67 & 70 & 76 \\
\hline $401-404$ & Hypertension & 355 & 277 & 294 & 311 & 339 \\
\hline $410-414$ & Ischaemic heart disease & 4700 & 3252 & 3561 & 3870 & 4350 \\
\hline $415-417$ & Pulmonary heart disease & 155 & 121 & 128 & 136 & 148 \\
\hline $420-429$ & Other heart disease & 1773 & 1359 & 1448 & 1536 & 1675 \\
\hline $430-438$ & Cerebrovascular disease & 1136 & 757 & 843 & 929 & 1079 \\
\hline 440 & Atherosclerosis & 79 & 61 & 64 & 68 & 75 \\
\hline 441 & Aortic aneurysm & 175 & 82 & 101 & 121 & 149 \\
\hline $442-448$ & Other arterial disease & 81 & 42 & 50 & 59 & 72 \\
\hline \multicolumn{7}{|c|}{ Adult pulmonary diseases } \\
\hline $480-487$ & Pneumonia and influenza & 491 & 339 & 371 & 403 & 451 \\
\hline $490-492$ & Bronchitis, emphysema & 214 & 40 & 75 & 110 & 158 \\
\hline 493 & Asthma & 73 & 53 & 57 & 62 & 69 \\
\hline 496 & Chronic airway obstruction, NOS & 627 & 114 & 217 & 320 & 457 \\
\hline \multicolumn{7}{|c|}{ Newborn and infant diseases } \\
\hline 765 & Short gestation/low birth weight & 341 & 298 & 310 & 336 & 350 \\
\hline 769 & Respiratory distress syndrome & 243 & 212 & 220 & 239 & 249 \\
\hline 770 & Respiratory conditions of the newborn & 252 & 220 & 228 & 248 & 258 \\
\hline 798 & Sudden infant death syndrome & 461 & 403 & 418 & 454 & 472 \\
\hline \multicolumn{7}{|c|}{ 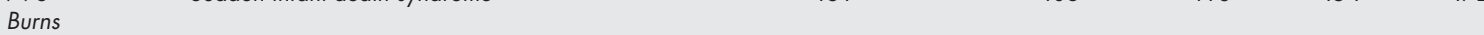 } \\
\hline $890-899$ & Burns & 49 & 0 & 0 & 0 & 0 \\
\hline \multicolumn{7}{|c|}{ Total YPLL85 } \\
\hline \multicolumn{2}{|c|}{ Scenario column sum } & 14702 & 8595 & 9901 & 11260 & 13263 \\
\hline \multicolumn{2}{|c|}{ Healthy People 2000 steady state prediction* } & & 11304 & 11810 & 12309 & 12429 \\
\hline \multicolumn{2}{|c|}{ Healthy People 2010 steady state prediction* } & & 10822 & 11399 & 11965 & 12106 \\
\hline
\end{tabular}


to two or three times the current prevalence of cigarette smoking. If nicotine accounts for $5 \%$ or less of cigarette smokers' risks, then a clean nicotine inhaler that displaces cigarettes results in much greater public health gains than Healthy People 2010 goals would produce, regardless of the prevalence of nicotine use. On average, eight nicotine users may be healthier than one smoker and seven non-smokers. For smokers and clinicians, the most attractive feature of a clean nicotine inhaler is that it could provide most of the benefits of smoking cessation to patients who are otherwise least likely to stop using deadly tobacco products. No other tobacco control policy offers this hope.

This analysis makes several assumptions unfavourable to the clean nicotine inhaler. First, the prevalence of nicotine use among pregnant women is assumed to equal that in young women. The model therefore exaggerates the deleterious perinatal effects of nicotine because some pregnant women will abstain from nicotine as they do from cigarettes. Second, DEMANDS assumes no changes in overall death rates with widespread nicotine inhaler use. Deaths avoided at an early age are redistributed according to currently observed death rates. In reality, avoidance of early smoking induced deaths should shift the distribution of all deaths later into life, resulting in greater benefits. Third, DEMANDS does not quantify never-smokers' mortality benefit from elimination of environmental tobacco smoke, which may total tens of thousands of YPLL85. This benefit would be larger in nicotine inhaler scenarios, which eliminate environmental smoke, than in the Healthy People 2010 scenario, which continues to expose some non-smokers to environmental smoke. The benefits of a clean nicotine inhaler that displaces cigarettes might be larger than this analysis predicts.

In spite of the general finding that public health might benefit equally from achieving Healthy People 2010 goals as from widely used, clean, fast acting nicotine inhalers, nicotine remains a drug deserving of some government regulation. Deeply inhaled nicotine may addict users just as efficiently as cigarettes. The decision making errors of youths and rationalisations of adults will persist. ${ }^{32}$ At the very least, consumers should know of nicotine's potential immunosuppressive effects, ${ }^{33}$ the possibility that nicotine is a gateway to drug and alcohol abuse, ${ }^{3435}$ and the high probability that nicotine permanently affects fetal brain development. ${ }^{36-43}$ Publicising these risks should dissuade many potential users, especially during pregnancy. If nicotine inhalers displace cigarettes without increasing overall nicotine use, then DEMANDS consistently predicts greater public health gains than Healthy People 2010 achievements would deliver.

\section{Making the switch}

Although public health gains might be similar, the efforts required to achieve Healthy People 2010 goals may be quite different than those required to displace cigarettes with clean nicotine. Achieving Healthy People goals may require indefinite public investment in intense smoking cessation and prevention interventions, as advocated by the Centers for Disease Control report on best tobacco control practices. ${ }^{44}$ Such efforts are difficult to initiate or maintain, as governments routinely divert funds from tobacco control. ${ }^{45}{ }^{46}$ If a novel, publicly funded tobacco control programme did dramatically reduce smoking it might also reduce tobacco tax revenues and become a net short term cost to the governments involved. Non-smoking voters seldom insist that their governments adhere to plans to spend tobacco tax revenue on tobacco control. These taxpayers may be even less likely to raise their own expenses to subsidise tobacco control. Furthermore, the formula for such dramatic success is elusive. It is not clear that the 2010 goal is achievable at all $^{47} 48$ or that all states or countries have the political will to achieve even the Healthy People 2000 goal of 15\% smoking prevalence. The Centers for Disease
Control reports that only Utah achieved that goal, with $12.9 \%$ of adults reporting smoking. ${ }^{49}$ Adult smoking was more common in California (17.8\%), Minnesota (19.8\%), Massachusetts $(20.0 \%)$, and Oregon $(20.8 \%)$, in spite of these states' tobacco control programmes. In 2002, $16.9 \%$ of US high school seniors smoked cigarettes daily, slightly below the previous nadir of $17.2 \%$ in $1992 .{ }^{50}$ While this is a welcome improvement over the mid 1990s, it would be premature to conclude that Healthy People 2010 goals are within reach.

In contrast, a tobacco control policy that includes a clean nicotine alternative to cigarettes requires legislative support, but less commitment of public resources and less innovation in tobacco control methods. The first policy step would be to allow the pharmaceutical industry to privately develop and market increasingly clean and fast acting nicotine delivery systems-for example, adapting an existing metred dose powder inhaler or adopting the design patented by Rose and colleagues. ${ }^{18}$ Policies to encourage substitution of nicotine inhalers for cigarettes would reflect established principles of community level tobacco control policy, such as raising tobacco product prices, informing consumers of risks, counter advertising, restricting youth access and marketing, and limiting opportunities to smoke. ${ }^{44}$ Taxation and product liability costs are already raising the price of cigarettes, and could create a significant price difference between cigarettes and less hazardous nicotine delivery systems. Governments should tax all nicotine delivery devices at rates that at least recover their regulatory and health costs. Governments would then tax safer nicotine products at a lower rate than hazardous tobacco products. ${ }^{6}$ Legislation could shield the nicotine and tobacco industries from liability for the health effects of nicotine use, on the theory that even addicted individuals bear some responsibility to weigh the known risks against the perceived benefits of nicotine, ${ }^{51}$ and in recognition of the historical futility of efforts to fully eradicate nicotine use. ${ }^{52}$ However, the same legislation should recognise that corporations could best anticipate, control, and manipulate perceptions of the harms inherent in their nicotine delivery systems. Legislation holding companies accountable for these harms could maintain higher liability costs for cigarettes than for clean inhalers. Labels on all nicotine products should inform consumers of risks, including addiction, and enumerate the products' chemical constituents. Product specific labels on cigarettes will list more chemicals and carry more warnings than the labels on clean nicotine inhalers.

As a special form of counter-advertising, legislation could permit promotion of clean nicotine delivery systems as an alternative to cigarettes, perhaps with fewer constraints than we apply to tobacco products. As with tobacco pipes and cigarette packages, the nicotine industry could produce myriad variations in the appearance of inhalers, so that users could select inhaler designs based on image. These images might even replicate successful smoking themes, such as rugged individuality, suave character, and pleasure. Pharmaceutical companies would then promote the images and real advantages of a modern nicotine inhaler to potential users, beginning with current smokers. Obviously, the government should continue to prohibit sales of all nicotine delivery systems, including clean inhalers, to young people. The government should monitor nicotine promotion in general, as corporations will wish to overstate the benefits of clean nicotine products. Local ordinances that curtail public smoking already create settings where a nicotine inhaler could be the most satisfying alternative for current smokers. Lacking a burning tip and side stream smoke, an inhaler should present no risk to bystanders, and requires no restriction on public use. Employers could even abolish outdoor smoking areas and smoking breaks if addicted employees could inhale nicotine indoors. Thus, nicotine inhalers would offer a valuable health benefit to current smokers, and rational policies based on established tobacco control principles could promote nicotine 
inhaler market share at the expense of cigarettes. These policies may be politically difficult to enact. Nevertheless, these policies require little public investment, might accelerate the contraction of the cigarette market, and should reduce or delay public health care costs to the extent that they promote inhaler use in place of cigarette smoking.

Some undesirable public health scenarios might unfold if a clean nicotine inhaler did not succeed in replacing tobacco cigarettes. First, the inhaler could simply disappear from the market, leaving the prevalence of cigarette use and nicotine addiction unchanged or even increased. Second, the inhaler could recruit new users, but not replace enough cigarette smoking to offset harms to the new users. However, even this short term public health failure would create legislative opportunities to demand lower nicotine content in cigarettes while raising cigarette taxes aggressively. ${ }^{63}$ Relatively satisfying inhalers could then supplant expensive and unsatisfying cigarettes and provide an alternative to black market cigarette purchases. $^{54}$

\section{Other potential nicotine hazards}

The net public health effect of allowing a clean, fast nicotine delivery system to compete with cigarettes is less certain than the obvious benefit of reducing all nicotine and tobacco use. Mathematical models would benefit from accurate estimates of risk attribution. The only certain approach to acquiring these data would require offering a palatable and inexpensive nicotine inhaler to a population of potential users (including never-smokers), then describing their experience. There may be no practical means of conducting a well controlled experiment of this sort.

However, we might be able to develop estimates of nicotine risks from other data. For example, smokeless tobacco users' relative risks for 26 SAMMEC 3 disease categories (excluding burns) provide an initial estimate for the sum of the risk fractions attributable to nicotine and correlates. Smokeless tobacco users' risks for many of these diseases may be available through analysis of existing data sets. Even slightly elevated risks of SAMMEC 3 diseases among smokeless tobacco users would deserve investigation before a policy shift that might increase use of nicotine in the general population.

Cardiovascular disease is a particular concern. Exposing large segments of the population to increased risks of ischaemic heart disease could overwhelm other health gains. As shown in table 1, SAMMEC 3 counts more YPLL85 from cardiovascular diseases than from any other cause. Consequently, small changes in risks for cardiovascular disease, especially ischaemic heart disease, substantially change DEMANDS predictions. However, nicotine would need to account for $30 \%$ or more of smokers' excess risk of cardiovascular diseases before DEMANDS would project a significant possibility of public health losses compared to 1990. This seems quite unlikely given the safety of nicotine replacement in the Lung Health Study, and among patients with established coronary artery disease..$^{55-59}$

Although nicotine replacement therapy is generally free of serious adverse consequences, ${ }^{60}$ nicotine might induce fatal diseases not anticipated by SAMMEC software. Nicotine causes a distinct acute toxicity syndrome typified by green tobacco sickness. ${ }^{61}{ }^{62}$ However, nicotine poisoning deaths are remarkably unlikely in tobacco harvesters, children ingesting nicotine or tobacco, ${ }^{63}$ or adults attempting suicide with nicotine patches. ${ }^{64}$ If nicotine use were to increase other substance abuse, then a clean nicotine inhaler could lead to increased deaths from alcohol and drug abuse. Current literature may not have explored complex pathways leading from nicotine use to human death. For instance, Heeschen and colleagues report that nicotine is angiogenic, ${ }^{65}$ and might promote tumour growth, but even this hypothetical harm might be offset by accelerated wound healing. ${ }^{66}$ Synergism

\section{What this paper adds}

Pharmaceutical grade nicotine replacement products are far safer than tobacco products, while novel tobacco products have uncertain risk profiles. The SAMMEC 3 (Smoking Attributable Morbidity, Mortality and Economic Costs) software from the US Centers for Disease Control and Prevention is a widely accepted model for estimating the number of deaths caused by cigarette smoking. However, SAMMEC 3 software cannot predict the health effects of a marketplace where cigarettes compete with, or are displaced by, potentially safer nicotine delivery systems.

A new computer program, DEMANDS (Differences in Expected Mortality Adjusted for Nicotine Delivery Systems), extends SAMMEC methods to estimate the health consequences of a marketplace where cigarettes compete with another nicotine delivery system. Adjustable DEMANDS inputs include the prevalence of smoking and other nicotine use, smoke and carbon monoxide delivery by the alternative system, and proportions of disease specific risks arising from nicotine, smoke, and carbon monoxide. For a wide range of plausible inputs, DEMANDS estimates that the health consequences of completely displacing cigarettes with a widely used, deeply inhaled, highly addictive, pharmaceutical grade nicotine inhaler are comparable or superior to reducing smoking prevalence to $12 \%$. Public health advocates and pharmaceutical companies could adapt tobacco control techniques to encourage smokers to replace cigarettes with nicotine inhalers. Nicotine inhalers might improve public health regardless of the political or economic fortunes of other tobacco control initiatives.

between nicotine and environmental risk factors other than cigarettes is conceivable. For example, chronic exposure to pure nicotine and polluted air might cause chronic obstructive lung disease.

DEMANDS does not estimate morbidity from nicotine use. Nicotine might impair quality of life or productivity by altering growth and function of the nervous system, with consequences potentially including depression, anxiety or addiction. ${ }^{8}$ These require further elaboration, separate analysis, and disclosure to smokers and other potential nicotine users.

Although a number of animal studies suggest negative health consequences for nicotine consumption, ${ }^{67-72}$ others seem to exonerate nicotine as a major cause of cardiopulmonary and other diseases. ${ }^{73-75}$ Modestly immunosuppressive nicotine could reduce an animal's resistance to infection by viruses, bacteria or mycobacteria. ${ }^{33}$ The effects of nicotine on fetal development need further investigation. ${ }^{76}$

\section{Limitations}

DEMANDS predicts consequences of nicotine use by extending the data and methods used in SAMMEC 3 software. DEMANDS therefore shares any inaccuracies present in SAMMEC 3 data or methods. SAMMEC 3 implicitly assumes that embedded relative risk data fairly represent smoking risks, and do not hide correlate risks. The relative risk data originate from the American Cancer Society Cancer Prevention Survey II, in which society volunteers chose families to follow for six years. ${ }^{77}$ Smoking correlates such as mental illness and poverty might be scarce in this population, reducing the need to estimate correlate risk fractions. Recent analyses suggest that relative risks for the most important tobacco related illnesses do not require adjustment for several potential correlate risks, including education, occupation, race, alcohol consumption, various dietary factors, hypertension, and diabetes. ${ }^{78} 79$ 
This report compares the consequences of steady state consumption of nicotine from one defined source, and therefore predicts the consequences of lifelong use of that source. The benefits of clean nicotine inhalers during a transitional period are more modest, because many users suffer from previous exposure to smoke. Likewise, in the year 2010, the immediate benefits of achieving Healthy People 2010 goals would be less dramatic than the benefits following decades of maintaining $12 \%$ smoking prevalence. Similarly, a clean nicotine inhaler that partially displaces cigarettes would confer more modest public health benefits.

\section{Conclusion}

Prevention of tobacco use is historically difficult in spite of clear health hazards. Regulatory responses to the problem are tenuous and subject to reversal or delay as political and economic fortunes change. A lasting reduction in tobacco related illness might result from unleashing clean alternative nicotine delivery systems to compete directly with tobacco products. Even if used very broadly, clean inhaled nicotine might reduce public health problems as much as a very successful tobacco control programme. Additional research should attempt to quantify the health consequences of using pure nicotine. Meanwhile, clinical and marketing trials of clean nicotine inhalers are defensible in populations with a high burden of smoking related illness.

\section{ACKNOWLEDGEMENTS}

I would like to thank Gary Giovino and anonymous reviewers for patient and astute critiques that greatly improved this paper.

\section{REFERENCES}

1 Benowitz N. Summary: risks and benefits of nicotine. In: Benowitz, ed. Nicotine safety and toxicity. New York: Oxford University Press, 1998: 185-95.

2 Institute of Medicine. Clearing the smoke: assessing the science base for tobacco harm reduction. Washington DC: National Academy Press, 2001

3 US Department of Health and Human Services. The health consequences of smoking: the changing cigarette. A report of the Surgeon General, 1981. Rockville, Maryland: Public Health Service, Office of the Assistant Secretary for Health, Office on Smoking and Health, 1981. (DHHS Publication No (PHS) 81-50156.)

4 Kozlowski LT, Strasser AA, Giovino GA, et al. Applying the risk/use equilibrium: use medicinal nicotine now for harm reduction. Tobacco Control 2001:10:201-3.

5 McNeil A, Foulds J, Bates C. Regulation of nicotine replacement therapies (NRT): a critique of current practice. Addiction 2001;96:1757-68

6 Warner KE, Slade J, Sweanor DT. The emerging market for long-term nicotine maintenance. JAMA 1997:278:1087-92.

7 Warner KE, Peck CC, Woosley RL, et al. Treatment of tobacco dependence: innovative regulatory approaches to reduce death and disease: preface. Food Drug Law J 1998;53(suppl): 1-8.

8 Slade J, Henningfield JE. Tobacco product regulation: context and issues. Food Drug Law J 1998:53(suppl):43-74.

9 Page JA. Federal regulation of tobacco products and products that treat tobacco dependence: are the playing fields level? Food Drug Law J 1998;53(suppl): $11-42$

10 Henningfield JE, Slade J. Tobacco-dependence medications: public health and regulatory issues. Food Drug Law J 1998;53(suppl):75-114.

11 Hoffmann D, Hoffmann I, El-Bayoumy K. The less harmful cigarette: a controversial issue. a tribute to Ernst L. Wynder. Chem Res Toxicol 2001;14:767-90.

12 Hoffmann D, Hoffmann I. The changing cigarette, 1950-1995. J Toxicol Environ Health 1997;50:307-64.

13 Gori GB. Low-risk cigarettes: a prescription. Science 1976;194:1243-6.

14 Stapleton JA, Russell MA, Sutherland G, et al. Nicotine availability from Eclipse tobacco-heating cigarette. Psychopharmacology (Berl) 1998;139:288-90.

15 Pierce JA, Gilpin EA. Impact of over-the-counter sales on effectiveness of pharmaceutical aids for smoking cessation. JAMA 2002;288:1260-4.

16 Schuh KJ, Schuh LM, Henningfield JE, et al. Nicotine nasal spray and vapor inhaler: abuse liability assessment. Psychopharmacology (Berl) 1997; 130:352-61.

17 Russell MA, Jarvis M, Sutherland G, et al. Nicotine replacement in smoking cessation. Absorption of nicotine vapor from smoke-free cigarettes. JAMA 1987;257:3262-5.

18 Rose J, Behm F, Turner J. 15 August 1995. USA patent 5441060.

19 Russell MA. The future of nicotine replacement. Br J Addict $1991 ; 86: 653-8$
20 Rodu B. An alternative approach to smoking control. Am J Med Sci 1994;308:32-4

21 Sweanor D. Regulatory imbalance between medicinal and non-medicinal nicotine. Addiction 2000;95(suppl 1):S25-8.

22 Eliasson B, Taskinen MR, Smith U. Long-term use of nicotine gum is associated with hyperinsulinemia and insulin resistance. Circulation 1996;94:878-81.

23 Lee TS, Hou X. Nicotine is hazardous to your heart. Chest 1996; 109:584-5

24 US Department of Health and Human Services. Healthy People 2010. Washington DC: Department of Health and Human Services, 2000.

25 CDC. Smoking-attributable mortality and years of potential life lost United States, 1988. MMWR Morb Mortal Wkly Rep 1991;40:62-3, 69-71

26 CDC. Cigarette smoking-attributable mortality and years of potential life lost - United States, 1990. MMWR Morb Mortal Wkly Rep 1993;42:645-9.

27 Nelson DE, Kirkendall RS, Lawton RL, et al. Surveillance for smoking-attributable mortality and years of potential life lost, by state United States, 1990. MMWR Morb Mortal Wkly Rep 1994;43:1-8.

28 Shultz JM, Novotny TE, Rice DP. Quantifying the disease impact of cigarette smoking with SAMMEC II software. Public Health Rep 1991:106:326-33.

29 Malarcher AM, Chrismon JH, Giovino GA, et al. Perspectives in disease prevention and health promotion smoking-attributable mortality and years of potential life lost - United States, 1984. MMWR Morb Mortal Wkly Rep 1997;46:444-51.

30 Thomas AR, Hedberg K, Fleming DW. Comparison of physician based reporting of tobacco attributable deaths and computer derived estimates of smoking attributable deaths, Oregon, 1989 to 1996. Tobacco Control 2001;10:161-4

31 Fellows JL, Trosclair A. Annual smoking-attributable mortality, years of potential life lost, and economic costs - United States, 1995-1999. MMWR Morb Mortal Wkly Rep 2002:51:300-3.

32 Hanson J, Kysar D. The joint failure of economic theory and legal regulation. In: Slovic $P$, ed. Smoking: risk, perception, and policy. Thousand Oaks, California: Sage Publications, Inc, 2001:229-76.

33 McAllister-Sistilli CG, Caggiula AR, Knopf S, et al. The effects of nicotine on the immune system. Psychoneuroendocrinology 1998;23:175-87

34 Haapanen A, Koskenvuo M, Kaprio J, et al. Carotid arteriosclerosis in identical twins discordant for cigarette smoking. Circulation 1989;80:10-16

35 Lindsay GB, Rainey J. Psychosocial and pharmacologic explanations of nicotine's "gateway drug" function. J Sch Health 1997;67:123-6.

36 Navarro HA, Seidler FJ, Schwartz RD, et al. Prenatal exposure to nicotine impairs nervous system development at a dose which does not affect viability or growth. Brain Res Bull 1989;23:187-92.

37 Navarro HA, Seidler FJ, Eylers JP, et al. Effects of prenatal nicotine exposure on development of central and peripheral cholinergic neurotransmitter systems. Evidence for cholinergic trophic influences in developing brain. J Pharmacol Exp Ther 1989;251:894-900.

38 Slotkin TA. Fetal nicotine or cocaine exposure: which one is worse? $J$ Pharmacol Exp Ther 1998;285:931-45.

39 Day NL, Richardson GA, Goldschmidt L, et al. Effects of prenatal tobacco exposure on preschoolers' behavior. J Dev Behav Pediat 2000;21:180-8.

40 Cornelius MD, Ryan CM, Day NL, et al. Prenatal tobacco effects on neuropsychological outcomes among preadolescents. J Dev Behav Pediatr 2001;22:217-25

41 Ernst M, Moolchan ET, Robinson ML. Behavioral and neural consequences of prenatal exposure to nicotine. J Am Acad Child Adolesc Psychiatry 2001;40:630-41.

42 Slotkin TA. The impact of fetal nicotine exposure on nervous system development and its role in suddent infant death syndrome. In: Benowitz $\mathrm{N}$, ed. Nicotine safety and toxicity. New York: Oxford University Press, 1998:89-97.

43 Oncken CA, Hardardottir H, Smeltzer JS. Human studies of nicotine replacement during pregnancy. In: Benowitz N, ed. Nicotine safety and toxicity. New York: Oxford University Press, 1998:107-116.

44 Centers for Disease Control and Prevention. Best practices for comprehensive tobacco control program - August 1999. Atlanta, Georgia: US Department of Health and Human Services, Centers for Disease Control and Prevention, Office on Smoking and Health, August 1999 .

45 Ritch WA, Begay ME. Smoke and mirrors: how Massachusetts diverted millions in tobacco tax revenues. Tobacco Control 2001;10:309-16.

46 Difranza JR, Rigotti NA. Impediments to the enforcement of youth access laws. Tobacco Control 1999;8:152-5.

47 Mendez D, Warner KE, Courant PN. Has smoking cessation ceased? Expected trends in the prevalence of smoking in the United States. Am J Epidemiol 1998;148:249-58

48 Mendez D, Warner KE. Smoking prevalence in 2010: why the healthy people goal is unattainable. Am J Public Health 2000;90:401-3.

49 Centers for Disease Control and Prevention. Tobacco control state highlights 2002: impact and opportunity. Atlanta, Georgia: Department of Health and Human Services, Centers for Disease Control and Prevention, National Center for Chronic Disease Prevention and Health Promotion, Office on Smoking and Health, 2002

50 Johnston L, O'Malley P, Bachman J. Teen smoking declines sharply in 2002, more than offsetting large increases in the early 1990s. Ann Arbor, Michigan: University of Michigan News and Information Services, 2002. 
51 Hilts P. Smokescreen: The truth behind the tobacco industry cover-up. Reading, Massachusetts: Addison-Wesley Publishing Co, 1996:30-37.

52 Kluger R. Ashes to ashes. New York: Alfred A Knopf, 1996:8-16.

53 Henningfield JE, Benowitz NL, Slade J, et al. Reducing the addictiveness of cigarettes. Council on Scientific Affairs, American Medical Association. Tobacco Control 1998;7:281-93.

54 Galbraith JW, Kaiserman M. Taxation, smuggling and demand for cigarettes in Canada: evidence from time-series data. J Health Econ 1997; 16:287-301.

55 Murray RP, Bailey WC, Daniels K, et al. Safety of nicotine polacrilex gum used by 3,094 participants in the lung health study. Lung health study research group. Chest 1996;109:438-45.

56 Benowitz NL, Gourlay SG. Cardiovascular toxicity of nicotine: implications for nicotine replacement therapy. J Am Coll Cardiol 1997;29:1422-31.

57 Benowitz N. Nicotine and cardiovascular disease. In: Benowitz N, ed. Nicotine safety and toxicity. New York: Oxford University Press, 1998:17-27

58 Rennard SI, Daughton D, Windle J. Toxicity of nicotine replacement in patients with cortonary artery disease In: Benowitz $\mathrm{N}$, ed Nicotine safety and toxicity. New York: Oxford University Press, 1998:49-53.

59 Wennmalm A. Cardiovascular effects of cigarette smoke and snuff. In: Benowitz N, ed. Nicotine safety and toxicity. New York: Oxford University Press, 1998:41-7.

60 Greenland S, Satterfield MH, Lanes SF. A meta-analysis to assess the incidence of adverse effects associated with the transdermal nicotine patch. Drug Safety 1998;18:297-308.

61 Hipke ME. Green tobacco sickness. South Med J 1993;86:989-92.

62 Ballard T, Ehlers J, Freund E, et al. Green tobacco sickness: occupational nicotine poisoning in tobacco workers. Arch Environ Health 1995;50:384-9.

63 CDC. Ingestion of cigarettes and cigarette butts by children - Rhode Island, January 1994-July 1996. MMWR Morb Mortal Wkly Rep 1997;46:125-8

64 Woolf A, Burkhart K, Caraccio T, et al. Self-poisoning among adults using multiple transdermal nicotine patches. J Toxicol Clin Toxicol 1996:34:691-8.
65 Heeschen C, Jang JJ, Weis $M$, et al. Nicotine stimulates angiogenesis and promotes tumor growth and atherosclerosis. Nat Med $2001 ; 7: 833-9$

66 Jacobi J, Jang JJ, Sundram U, et al. Nicotine accelerates angiogenesis and wound healing in genetically diabetic mice. Am J Pathol 2002;161:97-104.

67 Sayers NM, Drucker DB, Telford DR, et al. Effects of nicotine on bacterial toxins associated with cot death. Arch Dis Child 1995:73:549-51.

68 Chowdhury P, Doi R, Tangoku A, et al. Structural and functional changes of rat exocrine pancreas exposed to nicotine. Int J Pancreatol 1995; 18:257-64

69 Chowdhury P, Rayford PL, Chang LW. Induction of pancreatic acinar pathology via inhalation of nicotine. Proc Soc Exp Biol Med 1992:201:159-64.

70 Cluette-Brown J, Mulligan J, Doyle K, et al. Oral nicotine induces an atherogenic lipoprotein profile. Proc Soc Exp Biol Med 1986; 182:409-13.

71 Ashakumary L, Vijayammal PL. Effect of nicotine on lipoprotein metabolism in rats. Lipids 1997:32:311-5.

72 Silcox DH, 3rd, Daftari T, Boden SD, et al. The effect of nicotine on spinal fusion. Spine 1995;20:1549-53.

73 Waldum HL, Nilsen OG, Nilsen T, et al. Long-term effects of inhaled nicotine. Life Sci 1996;58:1339-46.

74 Syversen U, Nordsletten L, Falch JA, et al. Effect of lifelong nicotine inhalation on bone mass and mechanical properties in female rat femurs. Calcif Tissue Int 1999:65:246-9.

75 Loennechen JP, Nilsen OG, Arbo I, et al. Chronic exposure to carbon monoxide and nicotine: endothelin ET(A) receptor antagonism attenuates carbon monoxide-induced myocardial hypertrophy in rat. Toxicol Appl Pharmacol 2002; 178:8-14.

76 Dempsey DA, Benowitz NL. Risks and benefits of nicotine to aid smoking cessation in pregnancy. Drug Saf 2001;24:277-322.

77 Cancer Prevention Study II. The American Cancer Society Prospective Study. Stat Bull Metrop Insur Co 1992;73:21-9.

78 Malarcher AM, Schulman J, Epstein LA, et al. Methodological issues in estimating smoking-attributable mortality in the United States. Am J Epidemiol 2000;152:573-84.

79 Thun MJ, Apicella LF, Henley SJ. Smoking vs other risk factors as the cause of smoking-attributable deaths: confounding in the courtroom. JAMA 2000;284:706-12.

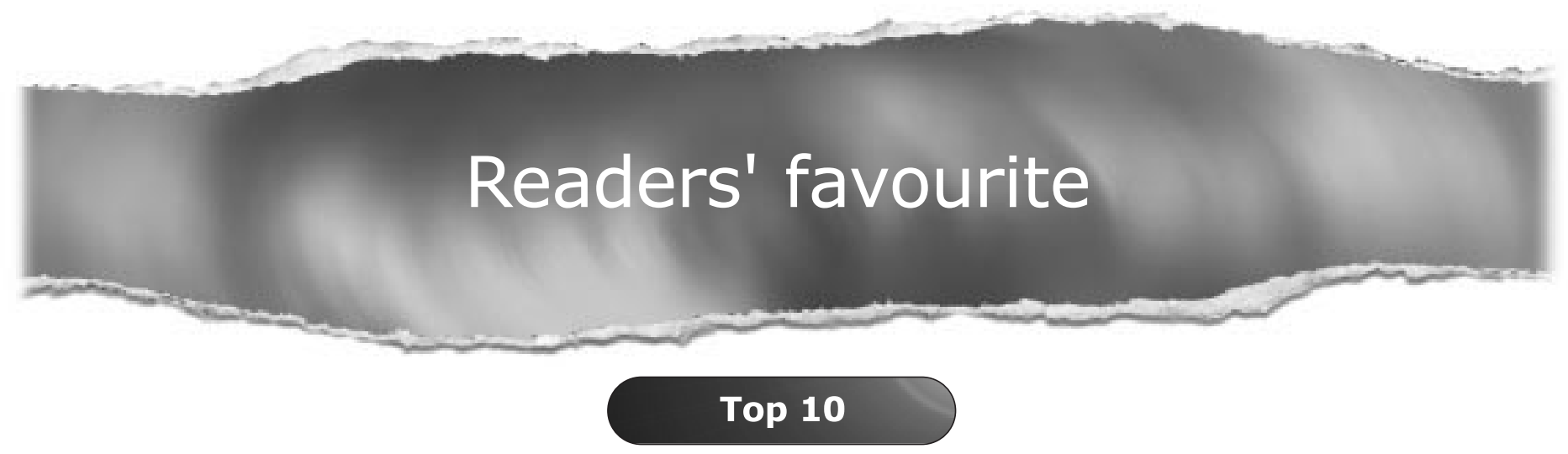

Click on the "Top 10" button on the homepage to see which are the best read articles each month

\section{www.tobaccocontrol.com}

